\title{
Transient expression of 'Candidatus Liberibacter asiaticus' effector SDE1 induces chlorosis by suppressing NbDDX3 gene in Nicotiana benthamiana
}

\section{Yinghui Zhou}

Fujian Agriculture and Forestry University

\section{Yanjiao Li}

Fujian Agriculture and Forestry University

Zhiqin Liu

Fujian Agriculture and Forestry University

Xuxu Zhao

Fujian Agriculture and Forestry University

Xianyuan Xu

Fujian Agriculture and Forestry University

\section{Yongping Duan}

USDA-ARS United States Horticultural Research Laboratory

Huasong Zou ( $\sim$ hszou@fafu.edu.cn )

\section{Research article}

Keywords: Candidatus Liberibacter asiaticus, Nicotiana benthamiana, SDE1, NbDDX3, chlorosis

Posted Date: March 28th, 2020

DOI: https://doi.org/10.21203/rs.3.rs-19409/v1

License: (c) (i) This work is licensed under a Creative Commons Attribution 4.0 International License. Read Full License 


\section{Abstract}

Background: 'Candidatus Liberibacter asiaticus' (Las) is the pathogenic bacterium that causes Huanglongbing in citrus plants, as well as in several types of experimental plants. Las releases a set of effectors to modulate host responses. One of these critical effectors is Sec-delivered effector 1 (SDE1), which induces chlorosis in Nicotiana benthamiana.

Results: Four SDE1-interacting proteins were identified from N. benthamiana, including DEAD-box RNA helicase DDX3, 26S proteasome non-ATPase regulatory subunit PSMD14, an ARM repeat protein, and a hypothetical protein. Gene silencing revealed that knockdown of the $N b D D X 3$ gene led to chlorosis in $\mathrm{N}$. benthamiana leaves. Fluorescent signal detection revealed that SDE1 was localized to the cell membrane, cytoplasm, and nucleus. Simultaneously, NbDD3 was expressed in cytoplasmic vesicles, as well as in the cell membrane. The interactions between SDE1 and NbDDX3 were shown to be localized on cell membrane using co-localization and bimolecular fluorescence complementation analysis. Moreover, the transcription of NbDDX3 gene was substantially suppressed in $N$. benthamiana plants that expressed SDE1.

Conclusion: Las effector SDE1 interacts with NbDDX3 at the cell membrane. Most importantly, the transient expression of SDE1 exerts a suppression effect on the transcription of $N b D D X 3$ gene. The silencing of $N b D D X 3$ leads to leaf chlorosis in $N$. benthamiana. This provided evidence to understand the molecular events in association with chlorosis induced by SDE1.

\section{Background}

Citrus Huanglongbing (HLB), also known as citrus greening, is one of the most destructive citrus diseases that causes severe losses in citrus production [3]. This disease has been associated with three species of phloemlimited pathogenic liberibacters that include Candidatus Liberibacter asiaticus (Las), Candidatus Liberibacter africanus, and Candidatus Liberibacter americanus [17]. The Las pathogen is highly prevalent and vectored naturally between citrus plants by insects, such as the Asian citrus psyllid, Diaphorina citri [3]. The liberibacters have been transferred to non-rutaceous hosts experimentally using Cuscuta (dodder). Plants that can serve as hosts include Catharanthus roseus (Garnier and Bové 1983), Solanum lycopersicum [7], Nicotiana tabacum [11] and Nicotiana benthamiana [30]. The characteristic disease symptoms include blotchy mottles on leaves and dieback of twigs. In addition, the fruits sometimes exhibit unusual coloration with undersized seeds, high acidity, and altered ripening dynamics [14].

Las-infected citrus plants exhibit a wide variety of types of physiological and biochemical transformation. After colonizing the plant, Las pathogen is disseminated to all tissues and organs within three months to one year [6]. Coupled with Las replication in the sieve tubes, HLB can be diagnosed by a high accumulation of starches, callose deposition, and multiple pockets of necrosis $[10,35]$. Since the photosynthetic genes are down-regulated, photoassimilated export is impaired in leaves $[23,26]$. The leaves that show chlorosis symptom are usually deficient in $\mathrm{N}, \mathrm{Fe}, \mathrm{Mg}$, and $\mathrm{Zn}[28,33]$. Moreover, HLB symptoms have been found to be closely related to a severe deficiency of phosphorus. In diseased citrus plants, a phosphorus deficiency induces a high level of expression of microRNA399. The exogenous application of phosphorus oxyanion solution significantly alleviates the severity of symptoms of HLB on citrus [41]. 
Bacterial pathogens deliver a number of proteins from their cytoplasm into the host cells and environment through specific protein secretion systems [1]. These secreted proteins are considered to have essential roles in increasing attachment, promoting bacterial virulence, and disrupting host defense reactions. The Las pathogen has been known to possess a general secretion system (Sec pathway) but without type II and type III secretion systems [8]. Eighty-six proteins with functional Sec-dependent secretion from Las were found to be secreted outside Escherichia coli cells using alkaline phosphatase fusion assays [32]. Many of these Sec-delivered effectors (SDEs) exhibit different expression levels in citrus and psyllids to manipulate the host processes [36, 38]. The effectors CLIBASIA_03915 and CLIBASIA_04250 induce phloem necrosis in senescent leaves of N. benthamiana [22]. The variations in symptoms on HLB-affected citrus plants are related to the genetic diversity of Las populations within two Las prophage regions [42]. The second prophage SC2 replicates as a prophage excision plasmid that encodes putative adhesin and peroxidase genes [40]. Transient expression of the peroxidase suppresses $\mathrm{H}_{2} \mathrm{O}_{2}$-mediated defense signaling pathway in citrus plants [18].

Sec-delivered effector 1 (SDE1) is a protein composed of 154 amino acids, and it can be secreted outside the cell through the Sec pathway upon expression in E. coli [32]. An SDE1-based polyclonal antibody has been successfully generated to diagnose and detect the bacterium owing to its specificity for Las pathogens [29]. The mature SDE1 protein without a signal peptide induces cell death in N. benthamiana in Agrobacterium-mediated transient transformation. In contrast, the full length SDE1 lacks the ability to induce cell death [29]. In addition, the deletion of a chloroplast targeting sequence resulted in starch accumulation and chlorosis but not cell death [30]. These findings indicate that SDE1 plays a pleiotropic role in inducing diverse host reactions, possibly by modulating different host target genes. The citrus papain-like cysteine proteases have been first characterized as SDE1-interacting proteins from hosts. However, their roles in HLB development remain unclear [4].

In this study, SDE1-interacting proteins were screened from N. benthamiana using a yeast two-hybrid $(\mathrm{Y} 2 \mathrm{H})$ technique. Their essential roles in SDE1-induced chlorosis were examined in gene silenced plants. These studies provide a detailed examination into the molecular events associated with SDE1-induced chlorosis, which will facilitate an understanding of the production of blotchy mottle phenotype of HLB.

\section{Results}

The full length SDE1 gene is not transcribed during Agrobacterium-mediated transient expression in N. benthamiana

The levels of expression of SDE1 were measured in N. benthamiana leaves subjected to Agrobacteriummediated transformation to determine the reason for the lack of induction of cell death during transient expression experiments of the full length SDE1 [31]. The coding sequences of full length SDE1 and mature SDE1 (SDE1 mp) were separately cloned into the binary vector pHB (Fig. 1a). The transient expression of SDE1 mp induced chlorosis in $\mathrm{N}$. benthamiana at 3 days post infiltration (dpi), while no chlorosis was observed in the infiltration area that transiently expressed the full-length SDE1 (Fig. 1b). Using EF1a as an internal control, quantitative RT-PCR (qRT-PCR) was conducted to evaluate the transcript levels of SDE1 and SDE1mp. The results showed that a high transcript level of SDE1 mp was detected. In contrast, the transcript level of SDE1 was extremely low (Fig. 1c). No transcript level of SDE1 was detected from uninoculated leaves or the leaves infiltrated with Agrobacterium that harbored empty vector pHB in all the qRT-PCR analyses (Fig. 1c). To determine the expression of SDE1 at protein level, SDE1 and SDE1mp were fused to the C-terminus of red 
fluorescent protein (RFP) in pGDR vector. At $2 \mathrm{dpi}$, a strong fluorescence signal was observed from $\mathrm{N}$. benthamiana cells that expressed the RFP-SDE1 mp fusion, and the signal was distributed in the cell membrane, cytoplasm, and nucleus (Fig. 1d). In contrast, no fluorescence was observed from the cells that expressed RFPSDE1 fusion (Fig. 1d). These results demonstrated that the full length SDE1 did not induce cell death in N. benthamiana merely because it could not be transcribed in Agrobacterium-mediated transient transformation. The mature protein SDE1 mp was used to test phenotypes in N. benthamiana in the following research.

Four SDE1-interacting proteins are screened from N. benthamiana

Using SDE1 as bait, a Y2H experiment was conducted to identify SDE1-interacting proteins from a N. benthamiana cDNA library. We obtained five positive clones, which corresponded to four $\mathrm{N}$. benthamiana genes. The duplicate clone was DEAD-box RNA helicase DDX3, while the other three included a 26S proteasome nonATPase regulatory subunit PSMD14, an ARM repeat protein Niben101Scf05290g02006.1, and unknown protein Niben101Scf04231g02014.1 (Table 1). The amino acids of four interactors shared more than $58 \%$ identity with their homologs in citrus species, including Citrus sinensis and C. clementine (Table 1). The pGADT7 plasmids recovered from yeast were co-transformed separately into AH109 cells using pGBKT7-SDE1. Each transformant was subjected to growth analysis on selective dextrose (SD) media SD/-Ade/-Leu/-Trp/-His/ and assayed for $\beta$ galactosidase to confirm the interaction (Fig. 2).

Table 1

The SDE1-interacting proteins of Nicotiana benthamiana and homologs in citrus plants

\begin{tabular}{|cllll|}
\hline \multirow{2}{*}{ No. } & Gene ID & Gene products & \multicolumn{2}{l|}{ Homologs in citrus plants } \\
\cline { 3 - 5 } & & & $\begin{array}{l}\text { Citrus sinensis } \\
\text { (Identity\%) }\end{array}$ & $\begin{array}{l}\text { Citrus clementine } \\
\text { (Identity\%) }\end{array}$ \\
\hline 1 & Niben101Scf02762g01005.1 & $\begin{array}{l}\text { DEAD-box ATP- } \\
\text { dependent RNA } \\
\text { helicase 3 }\end{array}$ & $\begin{array}{l}\text { orange1.1g011124m } \\
(70)\end{array}$ & $\begin{array}{l}\text { clementine0.9_002897 m } \\
(70)\end{array}$ \\
\hline 2 & Niben101Scf05290g02006.1 & $\begin{array}{l}\text { ARM repeat } \\
\text { protein }\end{array}$ & $\begin{array}{l}\text { orange1.1g005044m } \\
(81)\end{array}$ & $\begin{array}{l}\text { clementine0.9_003622 m } \\
(81)\end{array}$ \\
\hline 3 & Niben101Scf04231g02014.1 & Uncharacterized & $\begin{array}{l}\text { orange1.1g033279m } \\
(58)\end{array}$ & $\begin{array}{l}\text { clementine0.9_025591 m } \\
(58)\end{array}$ \\
\hline 4 & Niben101Scf02762g01005.1 & $\begin{array}{l}\text { DEAD-box ATP- } \\
\text { dependent RNA } \\
\text { helicase 3 }\end{array}$ & $\begin{array}{l}\text { orange1.1g011124m } \\
(70)\end{array}$ & $\begin{array}{l}\text { clementine0.9_002897 m } \\
(70)\end{array}$ \\
\hline 5 & Niben101Scf07364g00017.1 & $\begin{array}{l}\text { 26S } \\
\text { proteasome } \\
\text { non-ATPase } \\
\text { regulatory } \\
\text { subunit 14 }\end{array}$ & $\begin{array}{l}\text { orange1.1g032384m } \\
(99)\end{array}$ & $\begin{array}{l}\text { clementine0.9_016406 m } \\
\text { (99) }\end{array}$ \\
\hline
\end{tabular}

Silencing of NbDDX3 results in mottled leaves

To investigate the functions of four screened proteins, we created plants in which the respective transcripts were knocked down by virus-induced gene silencing (VIGS) using a tobacco rattle virus (TRV) vector. At $15 \mathrm{dpi}$, the 
NbDDX3-silenced plants exhibited yellow colors on the leaves, similar to the silencing of phytoene desaturase gene (PDS) in positive control plants (Fig. 3a). The mottled phenotype of NbDDX3-silenced plants was observed on the first three leaves after inoculation with a TRV construct. The late budding leaves on plants were completely bleached when they became mature. The NbPSMD14-silenced plants exhibited a severe developmental abnormality. At $15 \mathrm{dpi}$, new budding leaves immediately died (Fig. 3a). In contrast, the silencing of Niben101Scf05290g02006.1 or Niben101Scf04231g02014.1 genes did not lead to distinctive growth phenotypes. The silencing efficiency of each gene was evaluated using qRT-PCR analysis (Fig. 3b).

Examination of the chlorosis induced by SDE1 in gene-silenced plants

To determine whether the screened genes were involved in SDE1-mediated chlorosis, SDE1 and SDE1mp were transiently expressed in gene-silenced plants. Owing to the leaf death in silencing of NbPSMD14, those plants were not analyzed in this trial. Similar to the phenotype in wild type, full length SDE1 did not induce chlorosis on all gene-silenced plants. The transient expression of SDE1 $\mathrm{mp}$ induced chlorosis in both negative control and PDS-silenced plants (Fig. 4). The induction of chlorosis was difficult to discern on NbDDX3-silenced plants, since the leaves became mottled (Fig. 4). In the leaves from Niben101Scf05290g02006.1 or Niben101Scf04231g02014.1 gene-silenced plants, the transient expression of SDE1 mp induced a chlorotic phenotype similar to that found in wild type (Fig. 4).

Transient expression of SDE1 suppresses the transcription of NbDDX3

The phenotypes on NbDDX3-silenced plants encouraged us to determine the expression pattern of NbDDX3 in response to SDE1. Therefore, the transcript levels of NbDDX3 were measured in $\mathrm{N}$. benthamiana leaves that transiently expressed SDE1 $1 \mathrm{mp}$. The transcript level of NbDDX3 was reduced by $70 \%$ in plants that expressed SDE1 $\mathrm{mp}$ in comparison with the uninoculated control at $2 \mathrm{dpi}$ (Fig. 5). This demonstrated that the transcription of NbDDX3 gene was significantly suppressed when SDE1 mp was transiently expressed in N. benthamiana. It appeared that the chlorosis induced by SDE1 $\mathrm{mp}$ was caused by down-regulation of NbDDX3 transcription.

SDE1 Interacts With NbDDX3 At The Cell Membrane

The fused RFP-SDE1 $\mathrm{mp}$ was found to be localized on cell membrane, nuclei, and cytoplasm (Fig. 1d). A GFP$\mathrm{NbDDX} 3$ fusion was constructed to investigate its interaction with SDE1 $\mathrm{mp}$ in vivo. Fluorescent signal detection revealed that GFP-NbDDX3 accumulated to high levels in cytoplasmic vesicles with a small amount localized to the cell membrane (Fig. 6a). To examine the spatial interaction, GFP-NbDDX3 and RFP-SDE1 mp were transiently co-expressed in N. benthamiana cells. RFP-SDE1 $\mathrm{mp}$ was observed in the cell membrane, nucleus, and cytoplasm Nevertheless, the subcellular location of GFP-NbDDX3 was altered. In comparison with expression alone, a portion of NbDDX3 was located in the nucleus, with the exception of the cell membrane and organelles. Furthermore, an additional number of NbDDX3 proteins were recruited to the cell membrane. GFP-NbDDX3 and RFP-SDE1 mp were co-localized at the cell membrane and nucleus (Fig. 6b). A bimolecular fluorescence complementation assay was performed to further confirm the interaction between NbDDX3 and SDE1mp. This resulted in a clear yellow fluorescence that emitted from the cell membranes. In addition, the degrees of expression of SDE1 mp-YC and NbDDX3-YN were analyzed using a western blot (Fig. 6c). RFP-SDE1 mp was additionally co-expressed with GFP-XAC1347. GFP-XAC1347 is expressed in the membrane when transformed into N. benthamiana cells, implying that RFP-SDE1 mp was truly localized to the cell membrane (Supplementary 
Table 1). In this case, yellow fluorescence was observed from the cell membrane (Supplementary Fig. 1). These results suggested that a fraction of NbDDX3 interacts with SDE1 $\mathrm{mp}$ at the cell membranes.

\section{Discussion}

The transcript level of SDE1 gene was analyzed using qRT-PCR in Las-infected citrus, periwinkle, and psyllids in previous studies [29, 31]. Since a genetic manipulation system has not been established in Las, the secretion of SDE1 via Sec system was experimentally validated in E. coli cells [32]. In addition, diseased trees showed a positive signal against SDE1 polyclonal antibody [29]. These studies suggested that SDE1 functions as an effector during Las infection.

Various bacterial pathogens of plants are dependent on the type III secretion system to deliver virulence effectors into host cells [1]. These effectors were involved in the interference of host innate immunity, as well as mediating an incompatible interaction following recognition by plants [20]. Las is a phloem-limited bacterium with a small genome $(1.23 \mathrm{Mb})$ that does not possess the type III secretion system and related pathogenic effectors [8]. However, Las is transmitted directly into plant phloem cells through its insect vector in a manner similar to those of spiroplasmas and phytoplasmas [15]. Several effectors identified in phytoplasmas are transported to host cells via plasmodesmata before reaching the host cell cytoplasm $[2,16,37]$. Thereafter, SDE1 is proposed to be transported with the photosynthate transport flow [29].

The structural components of SDE1 were deciphered using a bioinformatics analysis. The N-terminal 24 amino acids represent a signal peptide, which is presumed to be cleaved by Las upon translocation [30]. The mature SDE1 without a signal peptide (SDE1 mp) induces cell death and chlorosis in N. benthamiana following Agrobacterium-mediated transient expression. However, the full length SDE1 does not induce any phenotype [30]. To obtain a more complete understanding of this difference, the expression of SDE1 was examined at mRNA transcriptional and protein translational levels. Our results showed that full length SDE1 was not transcribed during Agrobacterium-mediated transient transformation. This indicated that it was a failure of gene expression that precluded the full length SDE1 from causing chlorosis or cell death in $\mathrm{N}$. benthamiana. We assumed that, during Las infection, the plants are able to recognize SDE1 effector regardless of whether signal peptide is cleaved or not.

A previous study reported that SDE1 is localized to the vesicles surrounding the chloroplast and induces cell death in N. benthamiana [31]. With the exception of the signal peptide at $\mathrm{N}$-terminus, a chloroplast targeting sequence was found from SDE1 neighboring signal peptide. Deletion of the sequence converts cell death to the chlorosis phenotype [30]. By using a different fusion system, this study identified several subcellular localizations for RFP-SDE1 mp, including the cell membrane, protoplasm, and nuclei. In addition to the involvement of NbDDX3 in leaf chlorosis, the SDE1-interacting NbPSMD14 plays a role in growth of $\mathrm{N}$. benthamiana and could possibly be involved in SDE1-mediated cell death. These results support the concept that SDE1 targets several host genes to manipulate diverse plant responses.

RNA helicases are known to catalyze the unwinding of active and stable duplex RNA secondary structures in an ATP-dependent manner and have been generally observed in RNA metabolism $[5,25]$. Plant DEAD-box RNA helicases are involved in growth and development, as well as in the response to biotic and abiotic stresses [19, $39,43]$. Even though DEAD-box RNA helicases were first discovered from chloroplast-localized members, recent 
reports have demonstrated that plant DEAD-box RNA helicases are not limited to chloroplasts. Arabidopsis RNA helicase LOS4 is localized in the cytoplasm and enriched at nuclear rim [13]. Two stress response suppressors, STRS1 and STRS2, are localized in the nucleolus and nucleoplasm [21]. This study showed that N. benthamiana NbDDX3 was localized to the cell membrane with the exception of cytoplasmic vesicles. When NbDDX3 was coexpressed with SDE1, a number of NbDDX3 proteins were recruited to the cell membrane, which may facilitate its interaction with SDE1.

SDE1-interacting proteins were cloned from tobacco plants in this study because $\mathrm{N}$. benthamiana exhibits cell death and a chlorosis phenotype in response to SDE1 transient expression. In fact, N. benthamiana is a nonnatural host for Las even though it displays HLB symptoms similar to those of citrus hosts [30]. Fortunately, four SDE1-interacting proteins that have been cloned in this study have homologs in citrus plants. Further studies are needed to verify their functions in citrus hosts.

\section{Conclusions}

Four SDE1-interacting proteins have been identified from N. benthamiana. Gene silencing demonstrated that NbDDX3-silenced plants showed mottled leaves, which resemble the chlorosis phenotype induced by SDE1. These provide insights to characterize the key genes involved in chlorosis induced by SDE1.

\section{Methods}

Bacterial Strains and Plasmids

The plasmids and strains used in this study are listed in Supplementary Table 1. E. coli and A. tumefaciens strains were cultivated in Luria-Bertani media at $37^{\circ} \mathrm{C}$ and $28^{\circ} \mathrm{C}$, respectively. Yeast strain $\mathrm{AH} 109$ was cultured in YPD media ( $1 \%$ yeast extract, $2 \%$ peptone, and $2 \%$ glucose) at $30{ }^{\circ} \mathrm{C}$. Antibiotics were used at the following concentrations: Kanamycin (Km), $50 \mu \mathrm{g} / \mathrm{mL}$; Rifampicin (Rif), $50 \mu \mathrm{g} / \mathrm{mL}$; Ampicillin (Ap), 50 $\mu \mathrm{g} / \mathrm{mL}$; Spectinomycin (Sp), $25 \mu \mathrm{g} / \mathrm{mL}$.

DNA Manipulation And Plasmid Construction

DNA isolation, restriction enzyme digestion, and plasmid transformation were performed using standard methods [34]. The PCR primers used for molecular cloning and qRT-PCR analysis are listed in Supplementary Table 2.

To study the transcription levels in transient transformation, the coding sequences of full length SDE1 and signal peptide-deleted SDE1 mp were cloned separately into binary vector pHB at BamH I and Sac I sites. The coding sequence of SDE1mp was fused to C-terminus of RFP in pGDR at Sal I and BamH I sites to understand its subcellular localization and co-localization. NbDDX3 was fused to the C-terminus of GFP in pGDG. The coding sequences of SDE1 mp and NbDDX3 were cloned into 1301-YC and 1301-YN at Xba I and Kpn I sites, respectively, for bimolecular fluorescence complementation analysis. The resultant constructs were transformed into A. tumefaciens GV3101 using electroporation. A pGDG-XAC1347 construct was used for co-transformation with pGDR-SDE1mp to provide additional confirmation that the SDE1 protein is localized to cell membrane [9]. To isolate the interacting protein from $\mathrm{N}$. benthamiana, the coding sequence of SDE1 gene was cloned into 
pGBKT7 at EcoR I and Pst I sites to generate pGBKT7-SDE1. The alternative translation start codon GTG of SDE1 gene was replaced by ATG in forward primer (Supplementary Table 2).

Agrobacterium -mediated Transient Expression

A. tumefaciens strain GV3101 containing corresponding constructs was cultivated overnight in LB media. The cells were suspended in buffer $\left(10 \mathrm{mM} \mathrm{MgCl}_{2}, 10 \mathrm{mM} \mathrm{MES}\right.$, and $200 \mu \mathrm{M}$ acetosyringone, $\mathrm{pH}$ 5.7) to a final concentration of $\mathrm{OD}_{600}=0.2$. After $2 \mathrm{~h}$ of incubation, GV3101 cells were infiltrated into the leaves of $\mathrm{N}$. benthamiana grown in a growth room for 4 weeks at $25{ }^{\circ} \mathrm{C}$ with a $14 \mathrm{~h}$ light/ $10 \mathrm{~h}$ dark cycle. N. benthamiana leaves were collected at $2 \mathrm{dpi}$, and images were taken to detect the localization of fluorescent fusion protein using a Leica confocal laser scanning microscope (SP8, Leica, Wetzlar, Germany). Three different samples were examined under the microscope for each experiment. The phenotypes of $\mathrm{N}$. benthamiana induced by SDE1 and SDE1 mp were observed at $3 \mathrm{dpi}$. All the experiments were repeated four times.

qRT-PCR

RNA preparation and cDNA synthesis were performed as previously described [9]. qRT-PCR experiments were performed on a CFX Connect real-time system (Bio-Rad, Hercules, CA, USA) using iTaq Universal SYBR Green Supermix (Bio-Rad, Shanghai, China). The PCR thermal cycle conditions were as follows: denaturation at $95^{\circ} \mathrm{C}$ for $30 \mathrm{~s}$ and 40 cycles for $95^{\circ} \mathrm{C}, 5 \mathrm{~s} ; 58{ }^{\circ} \mathrm{C}, 20 \mathrm{~s}$. The expression of EF1a was used as an internal control. The relative expression level was determined and statistical analysis performed using CFX Maestro software (BioRad). The average threshold cycle was normalized according to the internal control in the Mode of Normalized expression $(\Delta \Delta \mathrm{Cq})$. Each experiment was repeated four times.

Y2H

A yeast two-hybrid assay was performed to screen SDE1-interacting proteins from a cDNA library of N. benthamiana. The initial Y2H screening was performed on SD/-Leu/-Trp/-His/ media using SDE1 as bait. pGADT7 plasmids were obtained from positive clones for DNA sequencing and a BLAST search. These plasmids were subsequently used to repeat the $\mathrm{Y} 2 \mathrm{H}$ experiment to confirm interaction with SDE1. The positive clones were cultivated on SD/-Ade/-Leu/-Trp/-His/ media supplemented with $20 \mu \mathrm{g} / \mathrm{mL}$ of X-a-galactosidase. The repeated $\mathrm{Y} 2 \mathrm{H}$ experiment was performed three times.

VIGS

The partial sequences of four SDE1-interacting genes were amplified by PCR and inserted into a pTRV2 vector. The resultant pTRV2 constructs were transformed into A. tumefaciens GV3101 using electroporation. A mixture of GV3101 cultures $(1: 1, \mathrm{v} / \mathrm{v})$ containing pTRV1 and pTRV2 constructs were co-infiltrated into 20-day-old N.

benthamiana leaves. The mixture of pTRV1 and pTRV2 empty vectors was used as a negative control, and the mixture of pTRV1 and TRV-PDS was used as a control for VIGS efficiency [24]. Agrobacterium-infiltrated plants were cultivated at $25^{\circ} \mathrm{C}$ using a $16 \mathrm{~h}$ light/ $8 \mathrm{~h}$ dark photoperiod. The transient expression of SDE1 was performed when new leaves had completely expanded. Each silencing experiment was repeated four times that included three independent plants.

Western Blot Analysis 
Total protein was extracted from N. benthamiana leaf disks using Laemmli buffer at 2 dpi [27]. The proteins were then resolved by $12 \%$ SDS-PAGE and subjected to immunoblot analysis. Anti-GFP was used to verify the expression of SDE1mp-YC, and anti-Myc was used to verify the expression of NbDDX3-YN fusion.

Sequence Analysis

A BLAST search was performed in the Sol Genomics Network (https://solgenomics.net/) to find N. benthamiana genes based on the CDNA sequence located in pGADT7. The homologs of four SDE1-interacting genes in C. sinensis and C. clementine were obtained from the Citrus Genome Database (https://www.citrusgenomedb.org/).

\section{Abbreviations}

Las : Candidatus Liberibacter asiaticus; SDE1: Sec-delivered effector 1; SDE1 mp: SDE1 mature protein; DDX: DEAD-box RNA helicase; PSMD: 26S proteasome non-ATPase regulatory subunit; HLB: Huanglongbing; qRT-PCR: Quantitative RT-PCR; dpi: Days post infiltration; RFP: Red fluorescent protein; GFP: Green fluorescent protein; Y2H: Yeast two-hybrid; TRV: Tobacco rattle virus; PDS: Phytoene desaturase gene; SD: Selective dextrose.

\section{Additional Files}

Additional file 1: Table S1. Bacterial strains and plasmids used in this study. Table S2. Primers for molecular cloning and qRT-PCR in this study. Figure S1. Colocalization of RFP-SDE1 and GFP-XAC1347 in N. benthamiana cells. Yellow fluorescence showing the colocalization in the cell membrane was indicated by arrows. Samples were examined under the microscope at $2 \mathrm{dpi}$. Scale bar denotes $50 \mu \mathrm{m}$.

\section{Declarations}

\section{Acknowledgements}

Not applicable.

\section{Authors' contributions}

Y. Z. performed the research; Y. L. and Z. L. analyzed the data; X. Z. and X. X. helped the bioinformatics analysis;

Y. D. and H. Z. wrote the manuscript.

\section{Funding}

This work was supported by the National Natural Science Foundation of China $(31671988,31872919)$.

\section{Availability of data and materials}

The datasets used and/or analysed during the current study are available from the corresponding author on reasonable request.

Ethics approval and consent to participate 
The authors declare that ethical problems are not applicable.

\section{Consent for publication}

Not applicable.

\section{Competing interests}

The authors declare that they have no competing interests.

\section{Author details}

${ }^{1}$ State Key Laboratory of Ecological Pest Control for Fujian and Taiwan Crops, College of Plant Protection, Fujian Agriculture and Forestry University, Fuzhou, 350002, China. ${ }^{2}$ U.S. Horticultural Research Laboratory, Agricultural Research Service, United States Department of Agriculture, Fort Pierce, FL, 34945, USA

\section{References}

1. Alfano JR, Collmer A. The type III (Hrp) secretion pathway of plant pathogenic bacteria: trafficking harpins, Avr proteins, and death. J Bacteriol. 1997;179:5655-

2. Bai X, Correa VR, Toruno TY, Ammar ED, Kamoun S, Hogenhout SA. AY-WB phytoplasma secretes a protein that targets plant cell nuclei. Mol Plant Microbe Interact. 2009;22:18-30.

3. Bové JM. Huanglongbing: A destructive, newly-emerging, century-old disease of citrus. J Plant Pathol. 2006;88:7-37.

4. Clark K, Franco JY, Schwizer S, Pang Z, Hawara E, Liebrand TWH., et al. An effector from the Huanglongbing-associated pathogen targets citrus proteases. Nat Commun. 2018;9:1718.

5. de la Cruz J, Kressler D, Linder P. Unwinding RNA in Saccharomyces cerevisiae. DEAD-box proteins and related families. Trends Biochem Sci. 1999;24:192-198.

6. Ding F, Duan YP, Paul C, Brlansky RH, Hartung JS. Localization and distribution of 'Candidatus Liberibacter asiaticus' in citrus and periwinkle by direct tissue blot immuno assay with an anti-OmpA polyclonal antibody. PLoS One. 2015;10:e0123939.

7. Duan YP, Gottwald TR, Zhou LJ, Gabriel DW. First report of dodder transmission of 'Candidatus Liberibacter a siaticus' to tomato (Lycopersicon esculentum). Plant Dis. 2008;92:

8. Duan YP, Zhou LJ, Hall DG, Li W, Doddapaneni H, Lin H, et al. Complete genome sequence of citrus huanglongbing bacterium, 'Candidatus Liberibacter asiaticus' obtained through metagenomics. Mol Plant Microbe Interact. 2009;22: 1011-1

9. Fan X, Guo J, Zhou Y, Zhuo T, Hu X, Zou H. The ColRS-regulated membrane protein gene XAC1347 is involved in copper homeostasis and hrp gene expression in Xanthomonas citri citri. Front Microbiol. 2018;9:1171.

10. Folimonova SY, Achor DS.Early events of citrus greening (Huanglongbing) disease development at the ultrastructural level. Phytopathology. 2010;100:949-58.

11. Francischini FJB, Oliveira KDS, Astúa-Monge G, Novelli A, Lorenzino R, Matiolli C, et al. First report on the transmission of 'Candidatus Liberibacter americanus' from citrus to Nicotiana tabacum Xanthi. Plant Dis. 
2007;91:631.

12. Garnier M, Bové JM. Transmission of the organism associated with citrus greening disease from sweet orange to periwinkle by dodder. Phytopathology.1983;73:1358-63.

13. Gong Z, Dong CH, Lee H, Zhu J, Xiong L, Gong D, et al. A DEAD box RNA helicase is essential for mRNA export and important for development and stress responses in Arabidopsis. Plant Cell. 2005;17:256-67.

14. Halbert SE, Manjunath KL. Asian citrus psyllids (Sternorrhyncha: Psyllidae) and greening disease of citrus: a literature review and assessment of risk in Florida. Florida Entomol. 2004;87:330-53.

15. Hogenhout SA, Oshima K, Ammar el-D Kakizawa S, Kingdom HN, Namba S. Phytoplasmas: bacteria that manipulate plants and insects. Mol Plant Pathol. 2008;9:403-23.

16. Hoshi A, Oshima K, Kakizawa S, Ishii Y, Ozeki J, Hashimoto M, et al. A unique virulence factor for proliferation and dwarfism in plants identified from a phytopathogenic bacterium. Proc Natl Acad Sci USA. 2009;106:6416-21.

17. Jagoueix S, Bové JM, Garnier M. The phloem-limited bacterium of greening disease of citrus is a member of the alpha subdivision of the Proteobacteria. Int J Syst Bacteriol. 1994;44:379-86.

18. Jain M, Fleites LA, Gabriel DW. Prophage-encoded peroxidase in 'Candidatus Liberibacter asiaticus' is a secreted effector that suppresses plant defenses. Mol Plant Microbe Interact. 2015;28:1330-7.

19. Jin H, Zhou L, Ge X, Zhang H, Zhang R, Wang C, et al. Cellular DEAD-box RNA helicase 18 (DDX18) promotes the PRRSV replication via interaction with virus nsp2 and nsp10. Virus Res. 2017;238:204-12.

20. Kay S, Bonas U. How Xanthomonas type III effectors manipulate the host plant. Curr Opin Microbiol. 2009;12:37-43.

21. Khan A, Garbelli A, Grossi S, Florentin A, Batelli G, Acuna T, et al. The Arabidopsis STRESS RESPONSE SUPPRESSOR DEAD-box RNA helicases are nucleolar-and chromocenter-localized proteins that undergo stress-mediated relocalization and are involved in epigenetic gene silencing. Plant J. 2014;79:28-43.

22. Li H, Ying X, Shang L, Redfern B, Kypraios N, Xie X, et al. Heterologous expression of CLIBASIA_03915/CLIBASIA_04250 by tobacco mosaic virus resulted in phloem necrosis in the senescent leaves of Nicotiana benthamiana. Int J Mol Sci. 2020;21:1414.

23. Liao HL, Burns JK. Gene expression in Citrus sinensis fruit tissues harvested from huanglongbing infected trees: comparison with girdled fruit. J Exp Bot. 2012;63:3307-3319.

24. Liu Y, Schiff M, Dinesh-Kumar SP. Tobacco Rar1, EDS1 and NPR1/NIM1 like genes are required for Nmediated resistance to tobacco mosaic virus. Plant J. 2002;30:415-29.

25. Lorsch JR. RNA chaperones exist and DEAD box proteins get a life. Cell. 2002;109:797-800.

26. Martinelli F, Uratsu SL, Albrecht U, Reagan RL, Phu ML, Britton M, et al. Transcriptome profiling of citrus fruit response to huanglongbing disease. PLoS One 2012;7:

27. Martinez-Garcia JF, Monte E, Quail PH. A simple, rapid and quantitative method for preparing Arabidopsis protein extracts for immunoblot analysis. Plant J. 1999;20:251-7.

28. Masaoka Y, Pustika A, Subandiyah S, Okada A, Hanundin E, Purwanto B, et al. Lower concentrations of microelements in leaves of citrus infected with 'Candidatus Liberibacter asiaticus'. Jpn Agric Res Q. 2011;45:269-75.

29. Pagliaccia D, Shi J, Pang Z, Hawara E, Clark K, Thapa SP, et al. A pathogen secreted protein as a detection marker for citrus Huanglongbing. Front Microbiol. 2017;8: 
30. Pitino M, Allen V, Duan YP. Las $\Delta 15315$ effector induces extreme starch accumulation and chlorosis as 'Candidatus Liberibacter asiaticus' infection in Nicotiana benthamiana. Front Plant Sci. 2018;9:

31. Pitino M, Armstrong CM, Cano LM, Duan YP. Transient expression of 'Candidatus Liberibacter asiaticus' effector induces cell death in Nicotiana benthamiana. Front Plant Sci. 2016;7:

32. Prasad S, Xu J, Zhang Y, Wang N. Sec-translocon dependent extracytoplasmic proteins of 'Candidatus Liberibacter asiaticus'. Front Microbiol. 2016;7:

33. Razi MF, Khan IA, Jaskani MJ. Citrus plant nutritional profile in relation to Huanglongbing prevalence in Pakistan. Pak J Agr Sci. 2011;48:299-304.

34. Sambrook J, Fritsch E F, and Maniatis T. 1989. Molecular cloning: a laboratory manual, 2nd ed. Cold Spring Harbor Laboratory Press, Cold Spring Harbor, NY.

35. Schneider H. Anatomy of greening-diseased sweet orange shoots.Phytopathology. 1968;58:1155-60.

36. Shi Q, Pitino M, Zhang S, Krystel J, Cano LM, Shatters RG Jr, et al. Temporal and spatial detection of Candidatus Liberibacter asiaticus putative effector transcripts during interaction with Huanglongbingsusceptible, -tolerant, and -resistant citrus hosts. BMC Plant Biol. 2019;19:122

37. Sugio A, Kingdom HN, MacLean AM, Griev EVM, Hogenhout SA. Phytoplasma protein effector SAP11 enhances insect vector reproduction by manipulating plant development and defense hormone biosynthesis. Proc Natl Acad Sci USA. 2011;108:1254-63.

38. Yan Q, Sreedharan A, Wei S, Wang J, Pelz-Stelinski K, Folimonova S, et al. Global gene expression changes in 'Candidatus Liberibacter asiaticus' during the transmission in distinct hosts between plant and insect. Mol Plant Pathol. 2013;14:391-404.

39. Zhang L, Yang Y, Li B, Scott IC, Lou X. The DEAD-box RNA helicase Ddx39ab is essential for myocyte and lens development in zebrafish. Development. 2018;145:

40. Zhang S, Flores-Cruz Z, Zhou L, Kang BH, Fleites LA, Gooch MD, et al. 'Ca. Liberibacter asiaticus' carries an excision plasmid prophage and a chromosomally integrated prophage that becomes lytic in plant infections. Mol Plant Microbe Interact. 2011;24:458-468.

41. Zhao H, Sun R, Albrecht U, Padmanabhan C, Wang A, Coffey MD, et al. Small RNA profiling reveals phosphorus deficiency as a contributing factor in symptom expression for citrus huanglongbing disease. Mol Plant. 2013;6:301-10.

42. Zhou LJ, Powell CA, Li WB, Irey M, Duan YP. Prophage-mediated dynamics of 'CandidatusLiberibacter asiaticus' populations, the destructive bacterial pathogens of citrus Huanglongbing. PloS One. 2013;8:

43. Zhu M, Chen G, Dong T, Wang L, Zhang J, Zhao Z, et al. SIDEAD31, a putative DEAD-box RNA helicase gene, regulates salt and drought tolerance and stress-related genes in tomato. PLoS One. 2015;10:

\section{Figures}


(a)

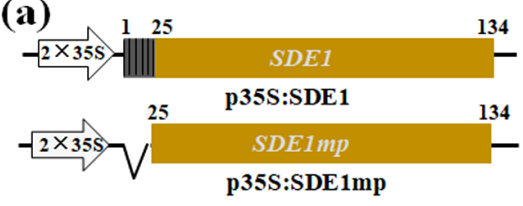

(b)

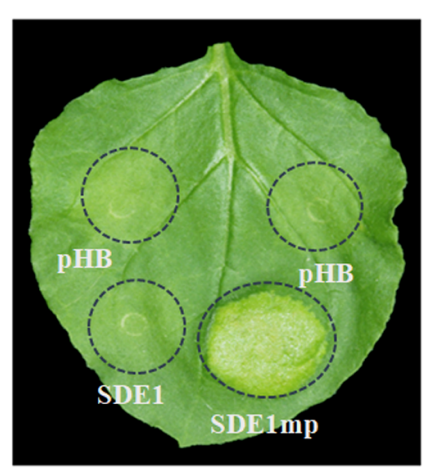

(c)

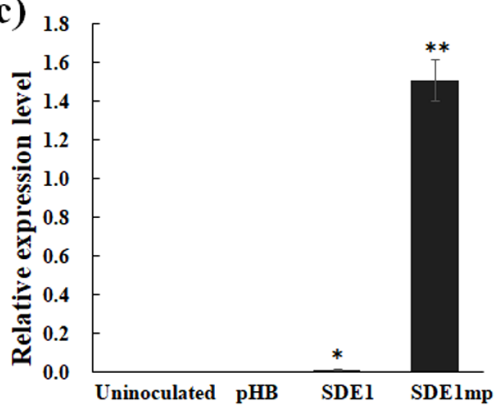

(d)
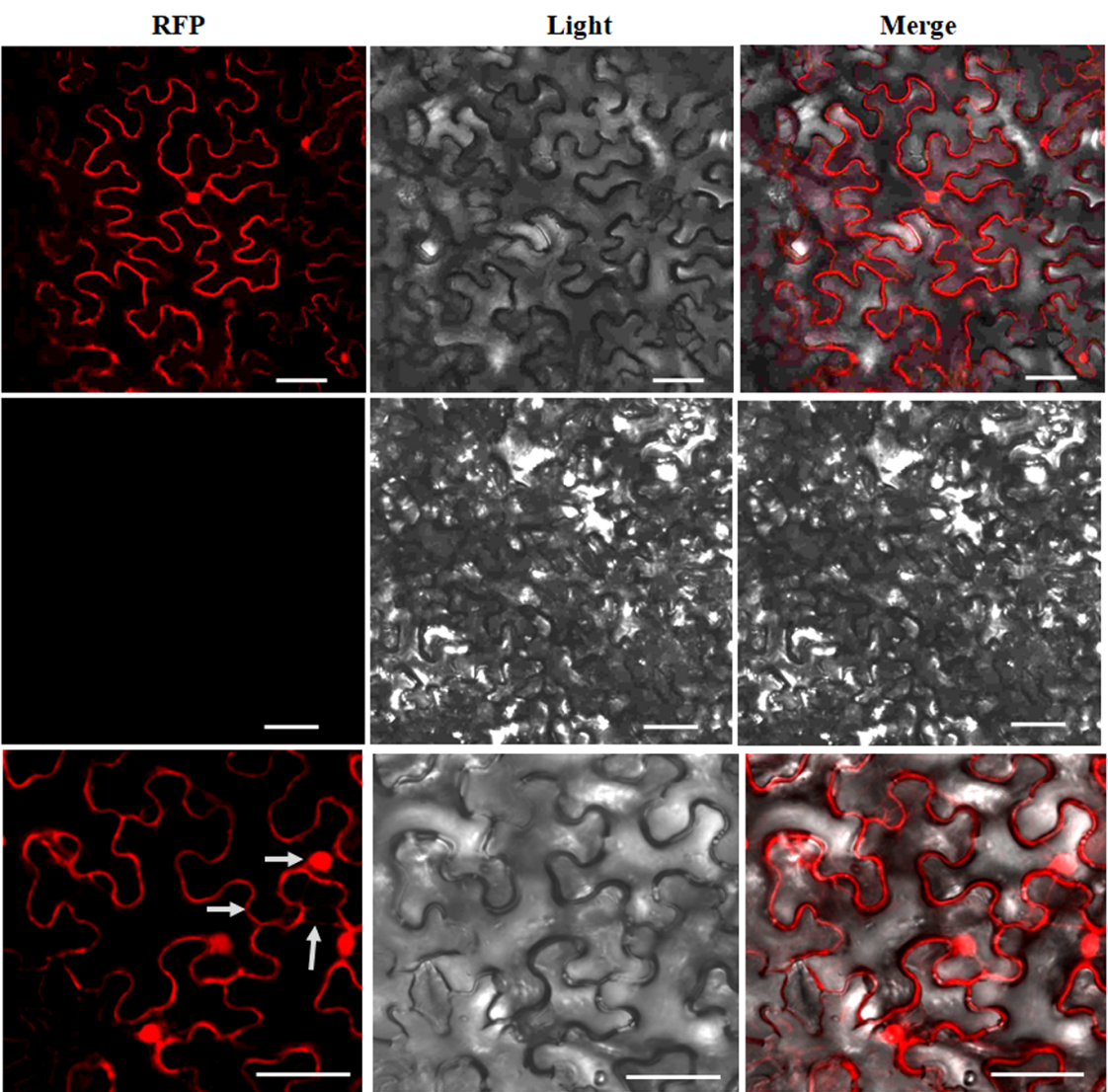

Figure 1

Expression of SDE1 in N. benthamiana by Agrobacterium-mediated transformation. a. Schematic diagram for full length SDE1 and mature SDE1 (SDE1 mp) for transient expression. The N-terminal 24 amino acids were deleted to generate SDE1mp. b. Chlorosis induced by SDE1 mp. The phenotype was observed at 3 dpi. c. qRTPCR assay of the transcript levels of SDE1 and SDE1 mp. The relative expression levels were normalized to internal control EF1a. Asterisks indicate significant differences $\left({ }^{*} P<0.05,{ }^{*} P<0.01, n=3\right)$. $d$. Visualization of RFP-SDE1 and RFP-SDE1mp fusion proteins in N. benthamiana cells. The localization of RFP-SDE1 mp in the cell membrane, cytoplasm, and nucleus is indicated by arrows. Samples were examined under the microscope at 2 dpi. Bar denotes $50 \mu \mathrm{m}$. 


\section{$\mathrm{OD}_{600}$}

\section{$\begin{array}{llll}1.0 & 10^{-1} & 10^{-2} & 10^{-3}\end{array}$}

\section{NbDDX3}

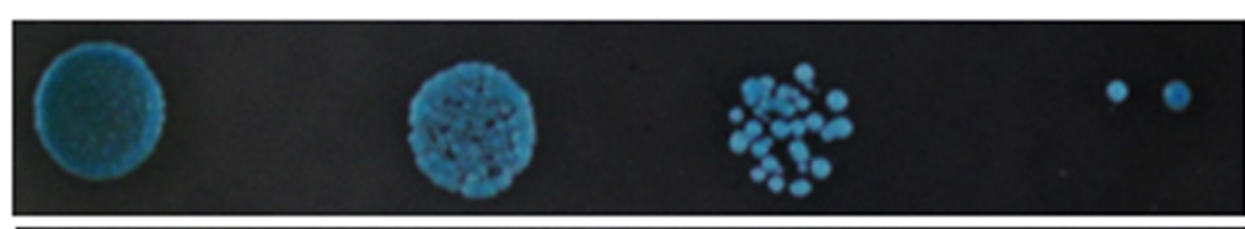

NbPSMD14

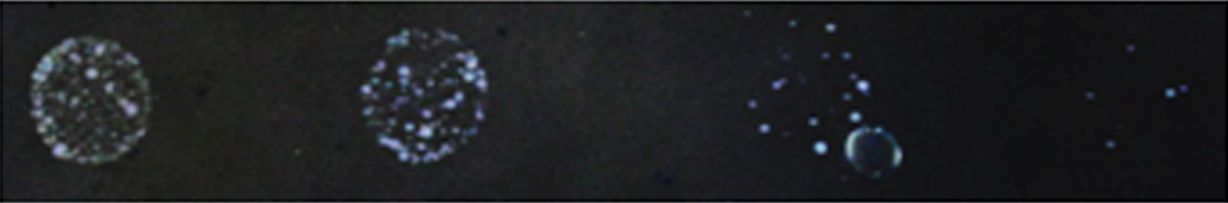

Niben101Scf05 290g02006.1

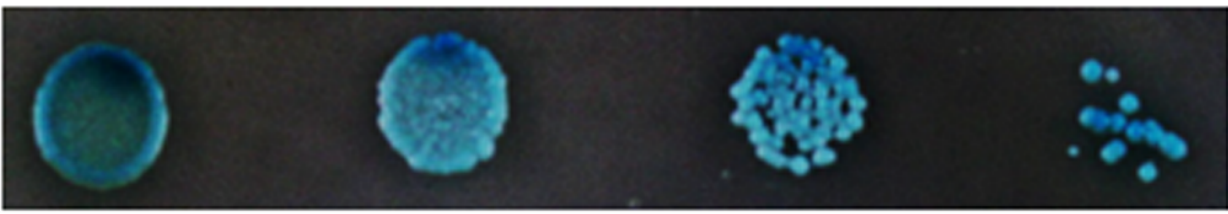

\section{Niben101Scf04 231g02014.1}

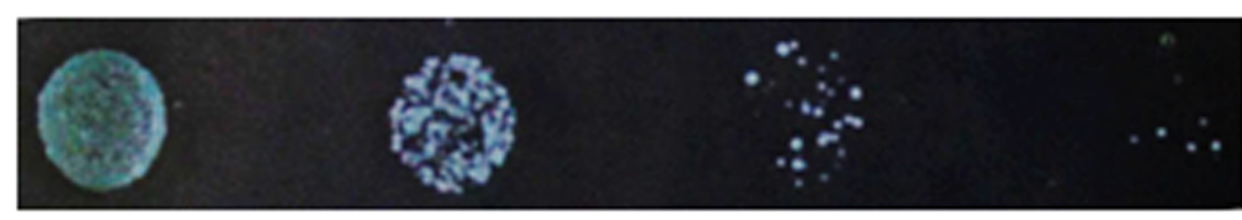
pGBKT7-SDE1

Figure 2

Screening of SDE1-interacting genes in N. benthamiana. AH109 transformants were grown on SD/-Ade/-Leu/Trp/-His media supplemented with $20 \mu \mathrm{g} / \mathrm{mL}$ X-a-galactosidase. 
(a)

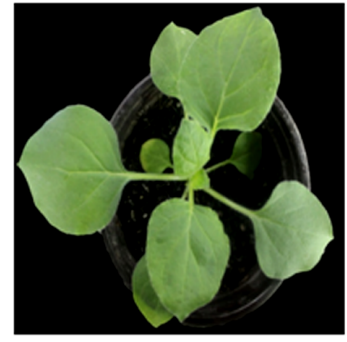

TRV:00

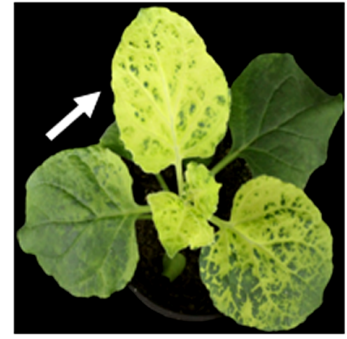

TRV:NbDDX3

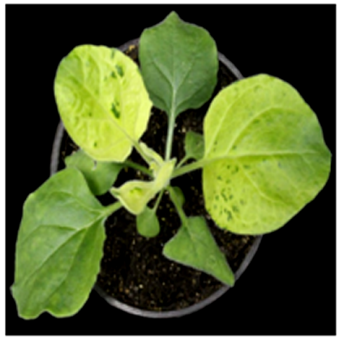

TRV:NbPDS

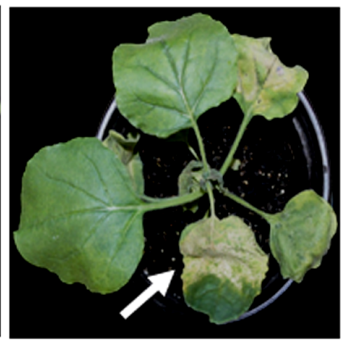

TRV:NbPSMDI4 (b)

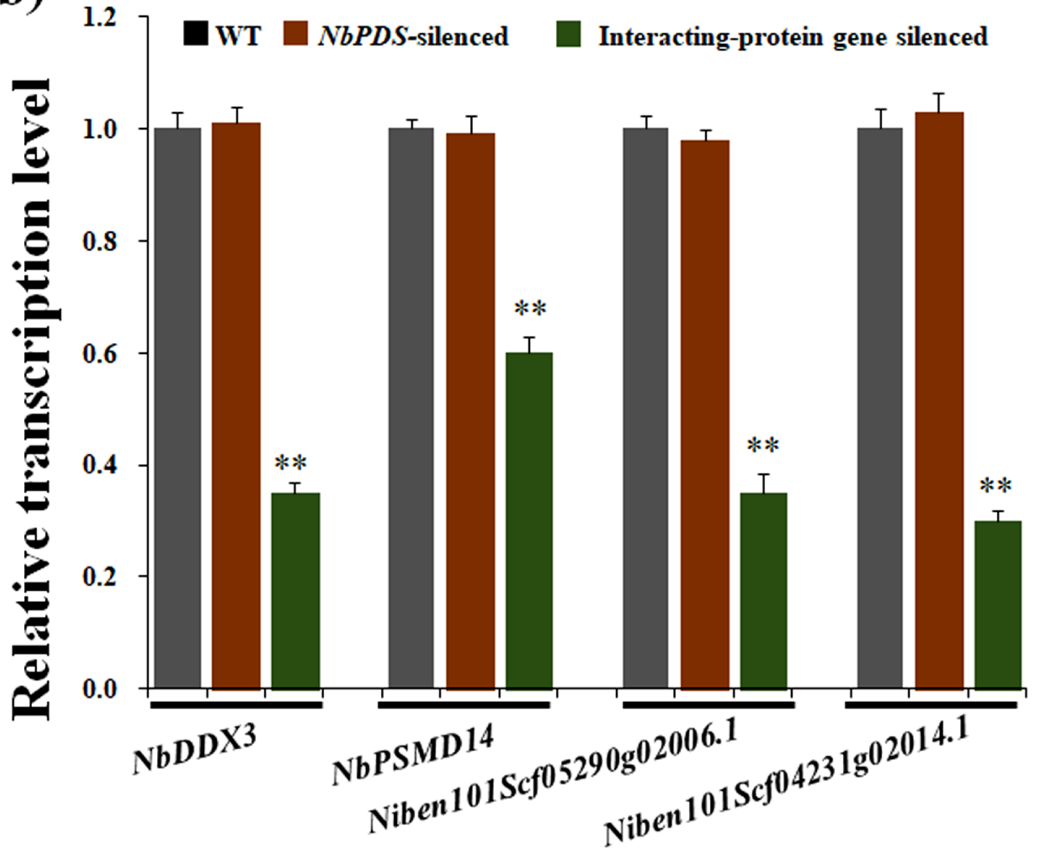

Figure 3

Silencing of SDE1-interacting genes in N. benthamiana. a. Growth phenotypes of NbDDX3- and NbPSMD14silenced plants. Mottling leaf in NbDDX3-silenced plant and dead leaf in NbPSMD14-silenced plant are indicated by white arrows. b. qRT-PCR analysis of the transcript level for each silenced gene. The expression of EF1a was used as internal control. The expression level of each gene in wild type plants was set to "1". The fold change in silenced plants was calculated by comparison with wild type. Error bars indicate standard deviations, and asterisks denote statistical significance (**P凶0.01, $n=3)$.

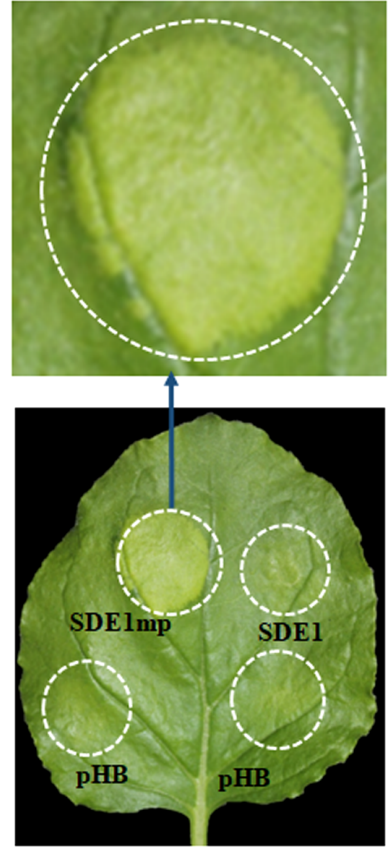

TRV:00
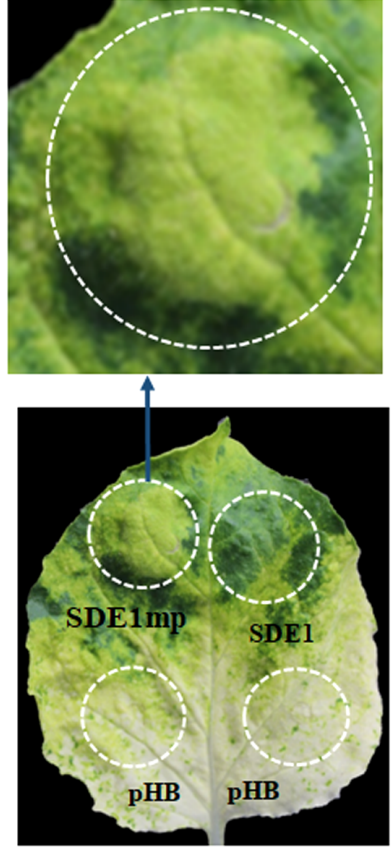

TRV:NbPDS
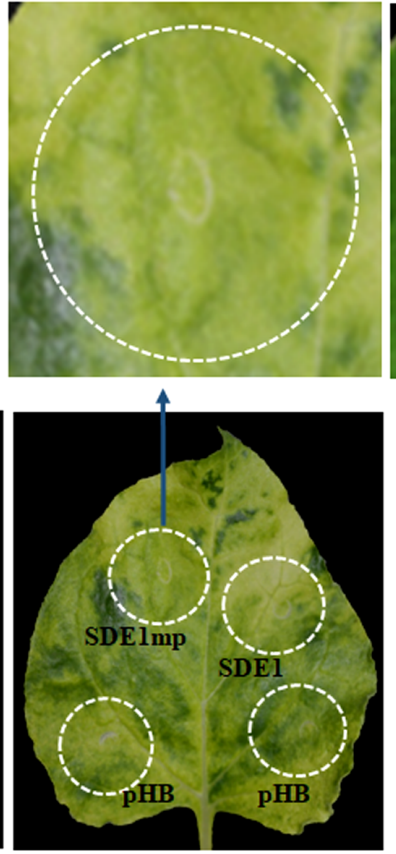

TRV:NbDDX3
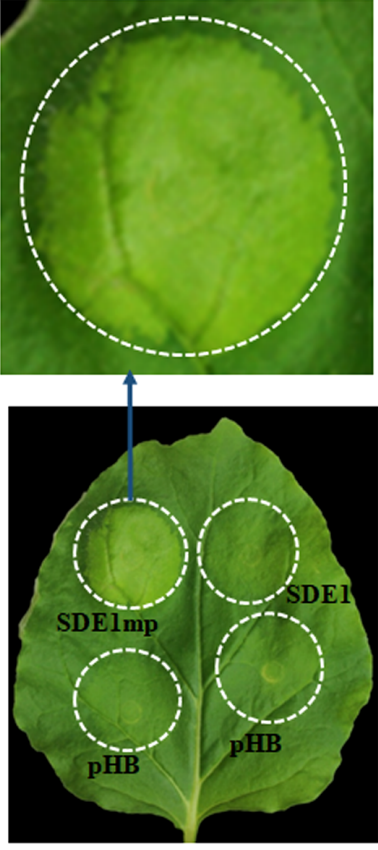

TRV:Niben101Scf05290g02006.1
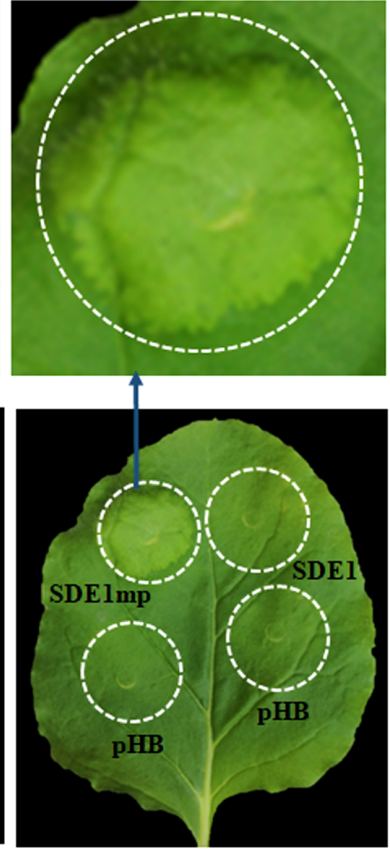

TRV:Niben101Scf04231g02014.1

Figure 4 
Examination of the chlorosis induced by SDE1 $\mathrm{mp}$ in gene silenced plants. The phenotype was recorded at $3 \mathrm{dpi}$. The infiltrated areas on each leaf are indicated by dotted circles.

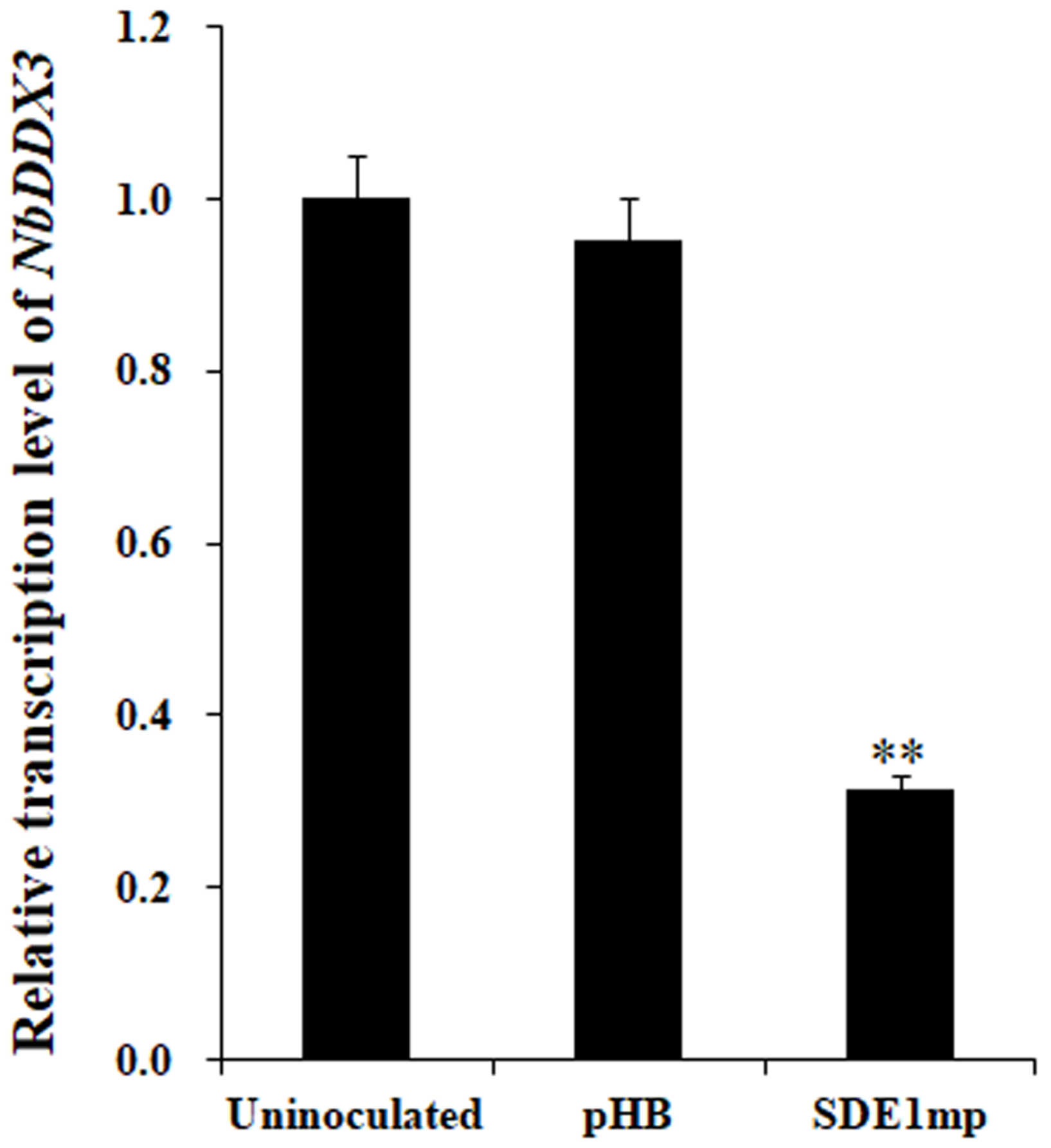

Figure 5

$\mathrm{NbDDX} 3$ transcript levels in $\mathrm{N}$. benthamiana plants that transiently expressed SDE1 mp. A qRT-PCR analysis was performed at $2 \mathrm{dpi}$. The expression of EF1a was used as an internal control. The level of expression of NbDDX3 in uninoculated plants was set to "1". The fold change in plants expressing SDE1 mp was calculated by comparison with uninoculated plants. Error bars indicate standard deviations, and asterisks denote statistical significance $(\star \star P 囚 0.01, n=3)$. 
(a)

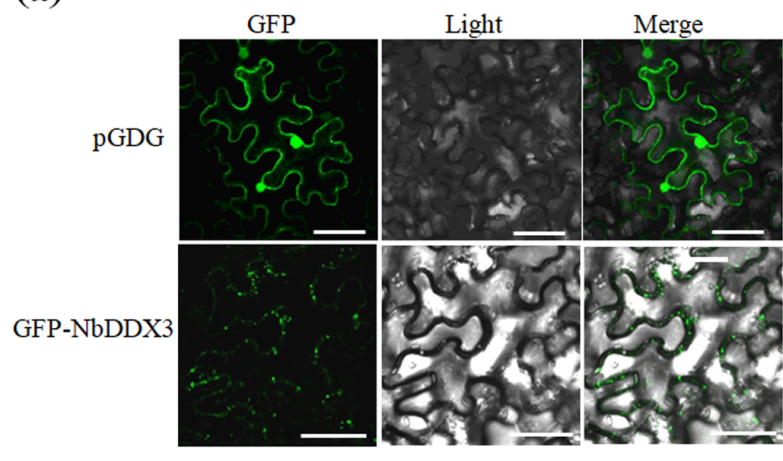

(b)

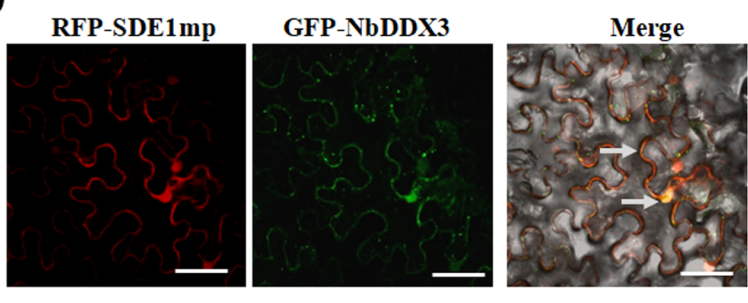

(c)

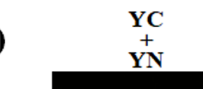

YC
$\stackrel{+}{\text { YN }}$

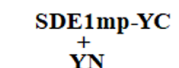

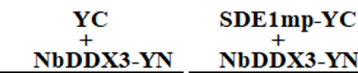

YFP

Light
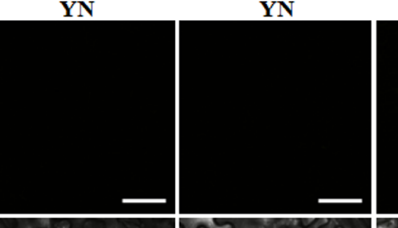

NbDDX3-YN
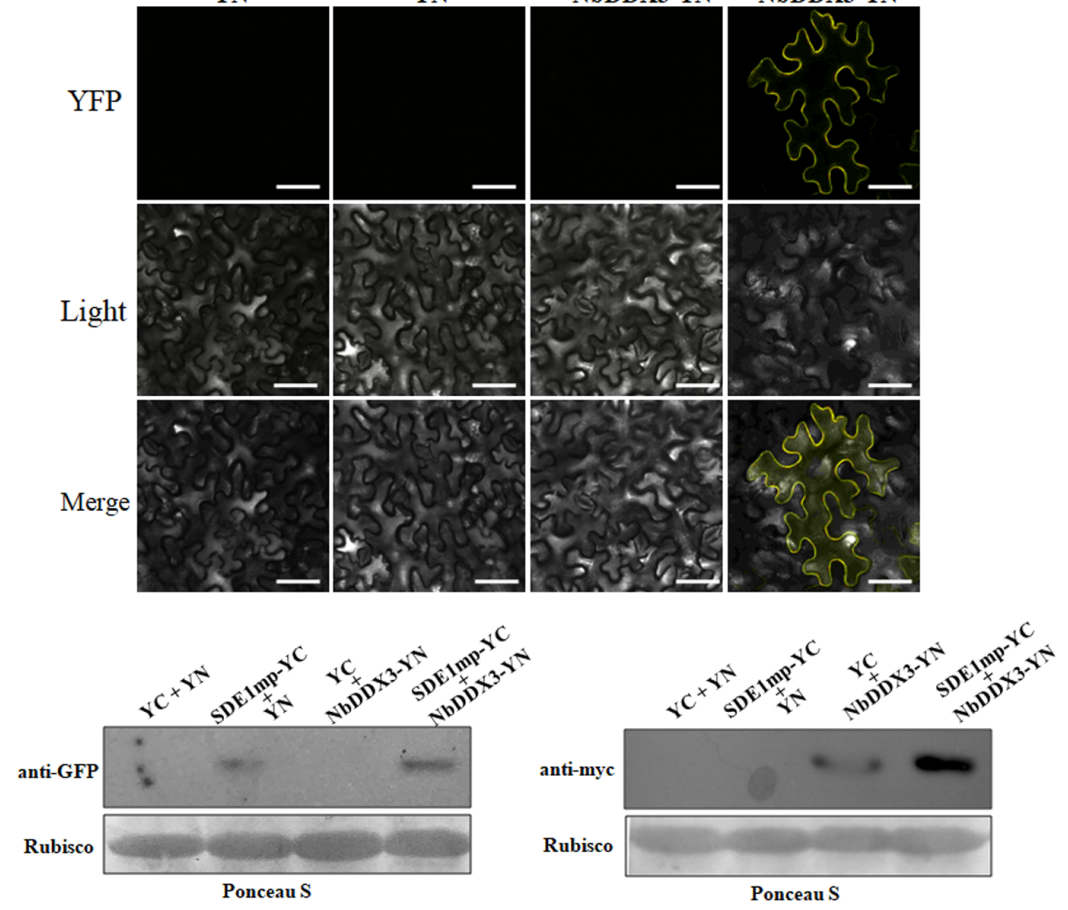

Figure 6

Subcellular localization of NbDDX3 and its interaction with SDE1 mp in N. benthamiana cells. a. Subcellular localization of GFP-NbDDX3 fusion proteins. b. Co-localization of RFP-SDE1mp and GFP-NbDDX3 on the cell membrane. c. Bimolecular fluorescence complementation analysis showing the spatial interaction of SDE1 and NbDDX3. Protein expression levels in the infiltrated plant leaves were collected for immunoblotting assays with anti-GFP and anti-Myc antibodies. Ponceau S staining indicates equal loading of the proteins. Samples were examined under the microscope at $2 \mathrm{dpi}$. Bar represents $50 \mu \mathrm{m}$.

\section{Supplementary Files}

This is a list of supplementary files associated with this preprint. Click to download.

- Fig.S1.tif

- SupplementaryTableS1.docx

- SupplementaryTables2.docx 